\begin{tabular}{|c|c|}
\hline 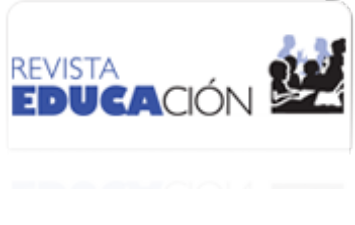 & $\begin{array}{l}\text { Revista Educación } \\
\text { ISSN: 0379-7082 } \\
\text { ISSN: } 2215-2644 \\
\text { revedu@gmail.com } \\
\text { Universidad de Costa Rica } \\
\text { Costa Rica }\end{array}$ \\
\hline
\end{tabular}

\title{
Aprendizaje basado en equipos en un curso de Ingeniería en Educación Superior
}

Reyes-Torres, Guillermo; H. Saavedra, Jorge; Aguayo-Vergara, Margarita

Aprendizaje basado en equipos en un curso de Ingeniería en Educación Superior

Revista Educación, vol. 44, núm. 1, 2020

Universidad de Costa Rica, Costa Rica

Disponible en: http://www.redalyc.org/articulo.oa?id=44060092026

DOI: https://doi.org/10.15517/revedu.v44i1.38316

Esta obra está bajo una Licencia Creative Commons Atribución-NoComercial-SinDerivar 3.0 Internacional. 


\title{
Aprendizaje basado en equipos en un curso de Ingeniería en Educación Superior
}

\author{
Team-Based Learning in a Higher Education Engineering Course
}

Guillermo Reyes-Torres

DOI: https://doi.org/10.15517/revedu.v44i1.38316

Escuela de Ingeniería Quimica de la Universidad del Bio-

Redalyc: http://www.redalyc.org/articulo.oa?id=44060092026

Bio, Chile

greyes@ubiobio.cl

(D) http://orcid.org/0000-0002-3468-1137

Jorge H. Saavedra

Escuela de Ingeniería Quimica de la Universidad del Bio-

Bio, Chile

jsaavedra@ubiobio.cl

(iD http://orcid.org/0000-0002-0007-7572

Margarita Aguayo-Vergara

Universidad del Bio-Bio, Chile

rmaguayo@ubiobio.cl

(iD) http://orcid.org/0000-0001-8204-9112

Recepción: 05 Agosto 2019

Aprobación: 18 Noviembre 2019

\section{RESUMEN:}

El Aprendizaje Basado en Equipos o por sus siglas en inglés conocido como: Team Based Learning (TBL), es una metodología de aprendizaje activa, donde el estudiantado construye el conocimiento a partir de tareas que desarrollan en equipos dinámicos, asumiendo un rol participativo. Esta metodología fue aplicada en estudiantes de pregrado del curso de Balances de Materia y Energía pertenecientes al programa de Ingeniería Civil Química de la Universidad del Bío-Bío, Chile. TBL fue implementado con el propósito de facilitar el trabajo en equipo, y mejorar el desempeño del estudiantado con respecto a las tradicionales clases magistrales. El trabajo fue desarrollado en la última unidad temática del curso para los años 2014 y 2016 y fue contrastado con los resultados alcanzados en los años 2015 y 2017, años en los cuales no se aplicó la metodología. Los resultados evidencian que las calificaciones finales promedio para el curso alcanzaron su valor más alto en el año 2016 (4.9), año en el cual se implementó la metodología por segunda vez. De igual manera las calificaciones en esta unidad fueron en promedio 6.5 y 6.6 para los años 2014 y 2016 frente a 6.0 y 4.9 para los años 2015 y 2017 respectivamente (en una escala de puntuaciones que tiene como valor mínimo 1.0 hasta un máximo de 7.0). Los resultados del cuestionario de percepción revelan que la metodología facilita el trabajo en equipo, formando un ambiente cooperativo de trabajo, donde más del $90 \%$ del estudiantado manifestó estar conforme con la metodología. Palabras Clave: Metodologías activas, Aprendizaje basado en equipos, Balances de materia y energía.

\section{ABstract:}

Team-Based Learning (TBL) is an active teaching method where students play an active role and learn through dynamic teambased tasks. This method was used by a group of undergraduate students in a Matter and Energy Balances course, part of the Chemical Civil Engineering Program at the University of Bío Bío in Chile. TBL was executed in order to facilitate teamwork and improve student performance as compared to a conventional lecture-based class. The study was conducted during the last topic unit of the course from 2014-2016, producing results that contrast those attained in 2015-2017. Based on the results, student grades peaked (4.9), achieving the highest final grade in 2016 (4.9) after implementing this method a second time. Average student grades were between 6.5 and 6.6 from 2014-2016 compared to 6.0 and 4.9 during 2015 and 2017 (based on a grading scale of 1.0 as a minimum and 7.0 as a maximum). The results of a perception questionnaire reveal that the method facilitates teamwork, creating a cooperative work environment where more than $90 \%$ of students feeling satisfied with the method.

KEYWORDS: Active Methodologies, Team-Based Learning, Mass and Energy Balances. 


\section{INTRODUCCIÓN}

Actualmente, la enseñanza centrada en el estudiantado ha promovido el uso de metodologías activas para transformar el proceso de enseñanza aprendizaje, en cuanto al rol docente y estudiante, el propósito de la formación y la evaluación. En este escenario, las metodologías activas se presentan como una herramienta de apoyo necesaria e indispensable para incorporar en las clases, creando una atmósfera adecuada para propiciar el aprendizaje (Felder, Brent y Carolina, 2009).

Una metodología activa no es más que un conjunto de actividades que cada estudiante ejecuta al interior del aula de clases, actividades en las cuales se involucra todo el grupo en la construcción del conocimiento. Las metodologías activas llevan al educando a una actitud proactiva, evitando la actitud pasiva que adopta el alumnado al reducir sus funciones a tomar notas en una clase expositiva en la cual solo participa de oyente, o en la cual responde simples preguntas que no llevan a un análisis a profundidad de las temáticas expuestas (Prince, 2004).

Existen diversas metodologías activas que han sido empleadas con éxito al interior de los cursos de Ingeniería (Bullard, Felder y Raubenheimer, 2008; Prince, 2004; Prince y Felder, 2006; Silverstein, Bullard y Vigeant, 2018), como por ejemplo es el caso de las metodologías activas STEM (acrónimo inglés para la combinación ciencia, tecnología y matemáticas) las cuales han mostrado un gran éxito al ser comparadas con las clases magistrales, demostrando un aumento de 1.5 veces la probabilidad de éxito en cursos de Ingeniería, Matemática y Ciencias (Freeman et al. 2014). Aun así, estas metodologías presentan importantes desafíos, dentro de los cuales el mayor desafío es la aplicación de estas a grupos grandes $n>50$ debido a que la estrategia y los recursos demandados se tornan complejos para grupos grandes (Freeman et al. 2014).

Por su parte la metodología de Aprendizaje Basado en Equipos (TBL) es utilizada ampliamente por docentes universitarios quienes han observado un mejor desempeño de sus estudiantes en la comprensión de los contenidos del curso, así como en el desarrollo del pensamiento crítico. De igual manera, se ha evidenciado una mayor asistencia y participación en las clases (Bullard et al. 2008; Freeman et al. 2014). Esta metodología requiere que el estudiantado sea estratégicamente organizado en grupos permanentes durante todo el período, para favorecer la diversidad y evitar coaliciones (Moraga y Soto, 2016).

Los contenidos de la asignatura son organizados en grandes unidades temáticas, cuatro en el caso de la asignatura de Balances de Materia y Energía. Previo al desarrollo de la clase, cada estudiante debe leer apuntes o documentos sobre algún contenido específico, facilitados por su docente, puesto que cada unidad se inicia con el Proceso de Aprendizaje Inicial [RAP] (Readiness Assurance Process) (Moraga y Soto, 2016).

El RAP es un examen corto sobre conceptos clave de la lectura, se aplica en forma individual y luego grupal, ocasión que tiene el alumnado de consensuar las respuestas. Al personal docente le corresponde retroalimentar inmediatamente el examen grupal, otorgando a sus estudiantes la oportunidad de elaborar apelaciones basadas en evidencia, cuando ellos perciben que pueden argumentar en forma válida sus respuestas erróneas. Para finalizar el RAP, se debe desarrollar una clase corta y específica, la cual permite al docente clarificar las dudas que pudieran haber surgido con posterioridad al examen grupal y a las apelaciones. Finalizado el RAP, se continúa con el desarrollo de actividades de aprendizaje, que permite al estudiantado aplicar los contenidos evaluados y planificados para la clase (Moraga y Soto, 2016; Tweddell, 2017). Resulta interesante destacar que el propósito del RAP no es solo motivar a estudiantes para que preparen sus clases, sino proporcionarles diversos momentos de retroalimentación sobre la comprensión del material facilitado por el cuerpo docente. La clave del éxito del RAP es proporcionar retroalimentación inmediata de su desempeño (Moraga y Soto, 2016).

En este contexto se propone una estrategia de innovación pedagógica, la cual involucra evaluaciones efectuadas por docentes y por estudiantes, bajo la tarea específica de desarrollar un blog que exponga un proceso industrial. Dicho trabajo es ejecutado por equipos integrados por estudiantes con diferentes estilos de aprendizaje. El objetivo principal es introducir al estudiantado de Ingeniería Química a una metodología 
activa de enseñanza aprendizaje, la cual les permita adquirir un rol protagónico en la construcción de conocimiento; a su vez que se fortalece la competencia de trabajo en equipo en el curso denominado Balances de Materia y Energía. La metodología de Aprendizaje Basado en Equipos fue aplicada en el año 2014 y 2016, y comparada con los resultados alcanzados sin su aplicación en los años 2015 y 2017 respecto a las calificaciones logradas por las/los estudiantes. Además, en los años 2014 y 2016 se recoge información relevante sobre la percepción de las/los estudiantes respecto a esta metodología.

\section{ANTECEDENTES PRÁCticos}

El concepto de Operaciones Unitarias puede ser un desafío en el aprendizaje por parte de estudiantes de los primeros años de la carrera de Ingeniería Civil Química, pues involucra la síntesis de diversos resultados de aprendizaje propios del ciclo de formación básico de los programas de Ingeniería Química (Silverstein et al. 2018). Este contenido exige que el estudiantado comprenda y elabore cálculos matemáticos en diferentes operaciones unitarias; se requiere, además, que se familiaricen previamente con los conceptos básicos de forma activa y permanente (Bullard y Felder, 2007); conceptos que pueden ser abordados mediante el estudio de casos simples, analogías y ejercicios prácticos. Sin embargo, en la práctica, estas herramientas didácticas suelen ser insuficientes, considerando que no favorecen una adquisición activa y efectiva del conocimiento, ni el desarrollo de habilidades cognitivas de nivel superior tales como: reflexión, argumentación, aplicación, evaluación y comparación. En este contexto una de las estrategias más adecuadas son las de aprendizaje cooperativo, donde cada estudiante, al construir conocimiento en equipo, explora, relaciona, codifica, compara y analiza problemas analíticos en el contexto, lo cual permite a la vez desarrollar el conocimiento, el procesamiento de la información y las habilidades críticas-reflexivas (Maure y Marimón, 2014). Es necesario Enseñar a pensar, para que el/la estudiante desarrolle capacidades y habilidades cognitivas que le ayuden al estudiantado a lograr el éxito en sus calificaciones, al mismo tiempo que se involucra de manera activa en su proceso de aprendizaje (Pérez y Poveda, 2008). El alumnado requiere establecer una analogía permanente entre conceptos teóricos y casos prácticos, de forma que no solo se obtiene conciencia de cómo y por qué se están aprendiendo los contenidos sobre operaciones unitarias, sino que también se pueda participar en el proceso de mejora continua al interior de esta temática (Bullard y Felder, 2007; Silverstein et al. 2018).

Existen varios estudios que muestran la importancia de la implementación de metodologías activas en la asignatura de Balances de Materia y Energía; que evidencian una mejora en el rendimiento académico del estudiantado, a diferencia del enfoque tradicional basado en la clase magistral, con evaluaciones enfocadas solo en la ejecución de exámenes escritos (Bullard y Felder, 2007; Keith, Silverstein, Visco y Bullard, 2010; Silverstein et al. 2018). Un método de evaluación acertado es también una razón más que motiva la búsqueda y el diseño de metodologías atractivas para el alumnado. Metodologías que, además, pueden incluir herramientas tales como: recursos tecnológicos, blogs en línea, evaluaciones por pares, entre otras. Dichas herramientas han mostrado efectividad en el logro de resultados de aprendizaje y aceptación por parte de las/ los estudiantes (Hemant y Stefanek, 2016; Liberatore, 2011; Mason, Shuman y Cook, 2013).

Enfrentarse al aprendizaje logrado desde el plano de la reflexión permanente es otro de los aspectos novedosos que incorpora el uso de los blogs (García, 2014; González, Montero y Laza, 2006; Zheng, Niiya y Warschauer, 2015). Es por ello, que han sido estudiados como herramienta educativa desde hace ya varios años y se han incorporados con bastante celeridad en los entornos educacionales debido a sus posibilidades de creación, participación y colaboración. 


\section{REFERENTES CONCEPTUALES}

El presente trabajo presenta una propuesta de innovación pedagógica, que combina el aprendizaje basado en equipos (TBL) con una herramienta tecnológica como es el blog (Tweddell, 2017); para lograr un cambio de paradigma desde la docencia centrada en el cuerpo docente centrada en el estudiantado en la asignatura de Balance de Materia y Energía.

La evaluación y evidencia de aprendizaje, consideran de manera adicional al blog virtual, la implementación de una coevaluación desarrollada por estudiantes para evaluar a sus compañeros de curso, y un cuestionario, en la cual se evalúa la percepción estudiantil respecto al trabajo desarrollado y del uso de la metodología TBL como una manera de mejorar la aplicación en contenidos futuros.

La implementación del estudio consideró cuatro fases que se visualizan en la Figura 1.

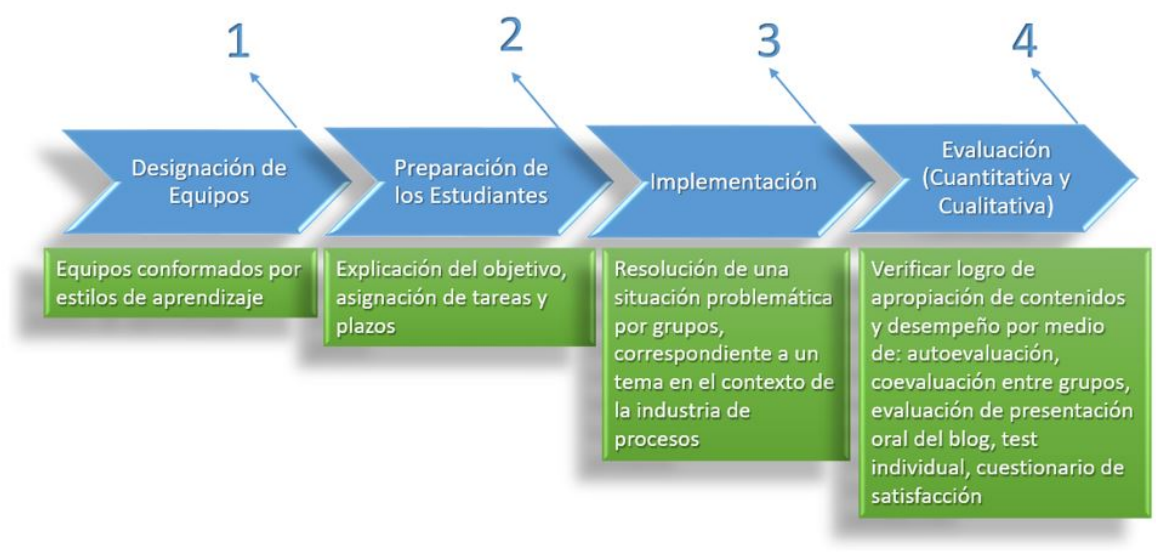

FIGURA 1

Fases de metodología TBL implementada para los años 2014 y 2016 Fuente: Elaboración propia

Tal como se observa en la Figura 1, el estudio se llevó a cabo en cuatro fases: designación de equipos, preparación estudiantes, implementación de la metodología y evaluación. Las evaluaciones incluyen un componente cuantitativo y otro cualitativo, el cual permite evaluar el nivel de adquisición de conocimientos y al mismo tiempo identificar el nivel de participación y aporte de todos los miembros de un equipo de trabajo.

\section{Procedimientos metodológicos}

\section{Tipo y diseño del estudio}

La investigación tuvo un carácter cuantitativo, en particular, se desarrolló un estudio exploratoriodescriptivo con un diseño transeccional. El objetivo fue observar y describir la incorporación de una metodología activa con uso de un recurso tecnológico, como es el blog en la formación de estudiantes. La investigación es exploratoria en cuanto se pretende dar una visión general de tipo aproximativo respecto al uso de metodologías activas de aprendizaje al interior del programa de Ingeniería Civil química de la Universidad del Bío-Bío, es descriptiva debido a que se busca especificar las características que tiene la implementación de la metodología, evaluando diversos aspectos tales como el rendimiento del estudiantado y su percepción respecto a la metodología. El diseño es transeccional debido a que se recolectan datos de la metodología de forma discreta en dos años puntuales (2014 y 2016). 


\subsection{Estructura de los grupos}

Esta experiencia pedagógica se enmarca en la carrera de Ingeniería Civil Química de una universidad chilena, específicamente en el curso denominado Balances de Materia y Energía, que se imparte en el cuarto semestre académico del programa de estudios con seis unidades del Sistema de Créditos Transferibles (SCT) equivalentes a nueve horas cronológicas de clase por semana o 14 horas pedagógicas de docencia directa por semana.

La metodología se aplicó en dos cursos independientes en los años 2014 y 2016 respectivamente, y como blancos de comparación se analizan dichos resultados frente al desempeño del estudiantado en los años 2015 y 2017 donde no fue implementada dicha metodología.

Se seleccionaron mediante muestreo no probabilístico 77 estudiantes ( 42 en el año 2014 y 35 en el año 2016). En la Tabla 1 se puede apreciar la composición por género y por año. El primer grupo del año 2014, constituido por seis grupos de cinco estudiantes y dos grupos de seis estudiantes. El segundo grupo (2016), constituido por cinco grupos de siete estudiantes. Para ambos grupos el promedio de edad de las/los estudiantes fue de 21 años. Con el fin de constituir grupos heterogéneos se integraron estudiantes con diferentes estilos de aprendizaje propuestos por (Alonso, Gallego y Honey, 2007; Honey y Mumford, 2000; Michaelsen, Knight y Fink, 2004). Los Estilos de Aprendizaje son los rasgos cognitivos, fisiológicos y afectivos, son indicadores, de cómo el alumnado percibe, interacciona y responde a los diferentes ambientes del aprendizaje, Según Alonso et al. (2007), es posible dividir los estilos en grandes características personales que se muestran en la Figura 2.

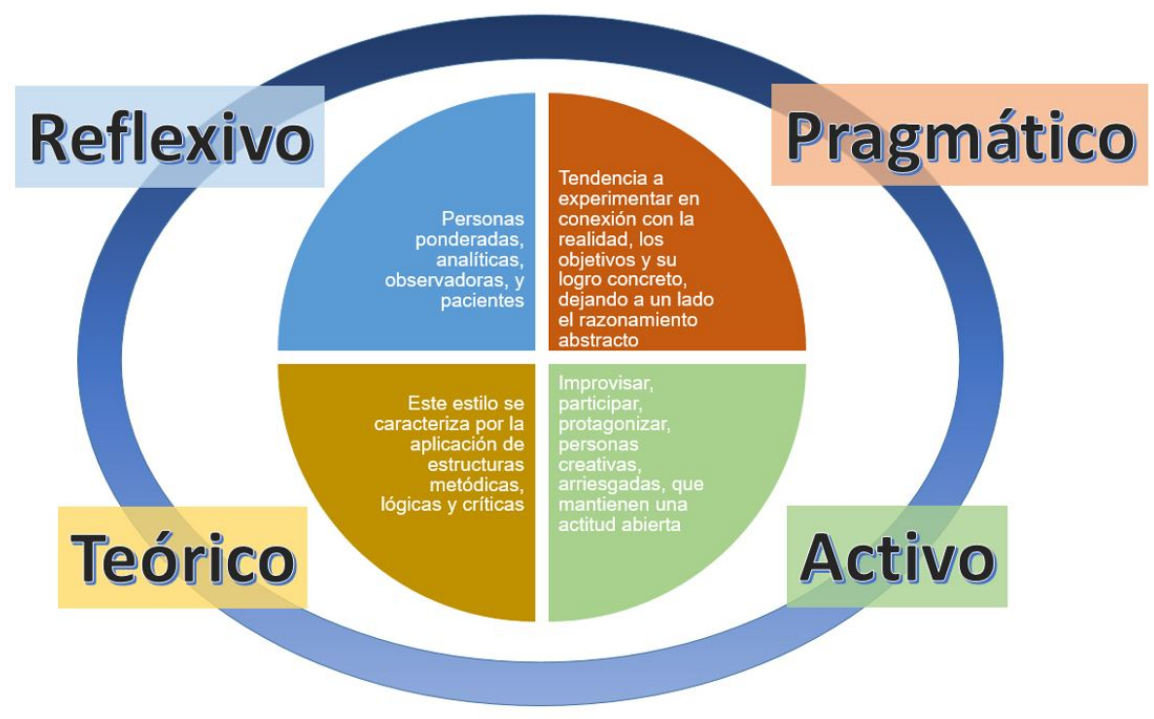

FIGURA 2

Descripción estilos de aprendizaje.

Fuente: Elaboración propia

Según los estilos de aprendizaje descritos anteriormente en la Figura 2 se consideró un cuestionario para conocer los hábitos de estudio de estudiantes, para así formar grupos heterogéneos constituidos por estudiantes que exhiben diferentes estilos y hábitos de aprendizaje. 
TABLA 1

Estructura de los grupos de trabajo (2014-2017)

\begin{tabular}{|c|c|c|c|c|c|c|c|c|}
\hline Metodología & Año & $\mathrm{M}$ & $\mathrm{H}$ & Total & PSA & $\begin{array}{c}\text { Desv. } \\
\text { PSA }\end{array}$ & $\mathrm{EP}$ & $\begin{array}{l}\text { Desv. } \\
\text { EP }\end{array}$ \\
\hline TBL & 2014 & $\begin{array}{l}11 \\
(26 \%)\end{array}$ & $\begin{array}{l}31 \\
(74 \%)\end{array}$ & 42 & 4.8 & $(0.5)$ & 21 & (2) \\
\hline Magistral & 2015 & $\begin{array}{l}27 \\
(44 \%)\end{array}$ & $\begin{array}{l}35 \\
(56 \%)\end{array}$ & 62 & 4.8 & $(0.4)$ & 21 & (1.5) \\
\hline TBL & 2016 & $\begin{array}{l}19 \\
(53 \%)\end{array}$ & $\begin{array}{l}16 \\
(47 \%)\end{array}$ & 35 & 4.8 & $(0.3)$ & 21 & (2.5) \\
\hline Magistral & 2017 & $\begin{array}{l}12 \\
(40 \%)\end{array}$ & $\begin{array}{l}18 \\
(60 \%)\end{array}$ & 30 & 4.7 & $(0.5)$ & 21 & (1.7) \\
\hline
\end{tabular}

Fuente: Elaboración propia

En la Tabla 1 se presenta la información para los años 2014 y 2016, años de aplicación de la metodología TBL, detallando la información por género $(\mathrm{M}=$ Mujeres, $\mathrm{H}=$ hombres), promedio semestral acumulado (PSA) y Edad Promedio (EP), estas dos últimas junto con sus respectivas desviaciones estándar (Desv.); se presenta adicionalmente la información correspondiente a los años 2015 y 2017 (años sin aplicación de la metodología). En la Tabla 1 se presenta información básica tal como género y edad, e información adicional sobre el promedio semestral acumulado (En escala de 1 a 7). Tal como se puede observar los grupos están constituidos en su mayoría por hombres (lo cual ha sido la tendencia de matrícula en el programa de Ingeniería Civil Química) con excepción del año 2016. En el año 2015 el curso contó con un gran número de estudiantes debido a que en ese año en particular a las/los estudiantes se les brindo de manera excepcional mayor flexibilidad en la cantidad de cursos que podían inscribir. En todos los demás aspectos tales como promedio semestral acumulado y edad, los grupos entre los años 2014 y 2017 son homogéneos no exhibiendo mayores diferencias.

\subsection{Actividad y su contexto}

La actividad fue desarrollada conforme a las etapas ilustradas en la Figura 1, designando y preparando los grupos de trabajo. La metodología no fue aplicada al curso en su totalidad de contenidos, fue aplicada solo en la cuarta y última unidad de contenidos, la cual bajo la lógica del modelo educativo de la Universidad del Bío-Bío no se conoce como unidad, sino como Resultado de Aprendizaje (RA). Los RA son una descripción explícita acerca de lo que una persona estudiante debe saber, comprender y ser capaz de hacer (Kennedy, Hyland y Ryan, 2014). Este último resultado de aprendizaje lleva por título: Modela procesos de la industria quimica y bioquímica, resolviendo balances de materia y energía acoplados, dicho resultado de aprendizaje corresponde a una síntesis del curso entero.

Una vez conformados los equipos, estos debieron resolver una situación problemática por grupos, correspondiente a un tema en el contexto de la industria de procesos. Se presentaron ocho situaciones modelo (ver Tabla S1 y S2 en material suplementario), de los cuales los grupos de estudiantes podían elegir uno o proponer otro similar, velando siempre por el cumplimiento de las siguientes condiciones:

-Corresponde a una situación real o a una problemática actual en el contexto de la industria de procesos.

-Existen dos o más alternativas a este proceso.

-Es posible desarrollar un análisis de balances de materia y energía sobre el o los procesos presentados.

-Es posible elaborar un análisis básico sobre el impacto ambiental, social y económico de los procesos estudiados. 


\subsection{Instrumentos}

Considerando que la evaluación es parte del desarrollo de competencias, la autoevaluación y la coevaluación son fundamentales para que cada estudiante se responsabilice de su proceso de aprendizaje. En este estudio, en lo referido al diseño pedagógico, se utilizaron: autoevaluaciones y coevaluaciones grupales e individuales, presentación oral del blog, y test individual de conocimientos. El detalle de las evaluaciones y sus ponderaciones se muestran en la Tabla 2. 
Guillermo Reyes-Torres, et al. Aprendizaje basado en eQuipos en un Curso de Ingeniería en Educació...

TABLA 2

Evaluaciones y sus ponderaciones

\begin{tabular}{|c|c|c|c|}
\hline $\begin{array}{l}\text { Evaluación y } \\
\text { abreviación }\end{array}$ & $\begin{array}{l}\text { Finalidad } \\
\text { del } \\
\text { instrumento }\end{array}$ & Objetivo & $\begin{array}{l}\% \\
\text { Nota }\end{array}$ \\
\hline $\begin{array}{l}\text { Autoevaluación } \\
\text { grupal (AG) }\end{array}$ & $\begin{array}{l}\text { Las/los } \\
\text { estudiantes } \\
\text { acuerdan } \\
\text { una } \\
\text { calificación } \\
\text { para su } \\
\text { propio } \\
\text { equipo de } \\
\text { acuerdo a la } \\
\text { percepción } \\
\text { de objetivos } \\
\text { alcanzados, } \\
\text { siguiendo la } \\
\text { rúbrica } \\
\text { entregada }\end{array}$ & $\begin{array}{l}\text { Incentivar una } \\
\text { percepción } \\
\text { autocrítica } \\
\text { frente al } \\
\text { desempeño del } \\
\text { trabajo en } \\
\text { equipo }\end{array}$ & $10 \%$ \\
\hline $\begin{array}{l}\text { Coevaluación } \\
\text { entre grupos } \\
\text { (CG) }\end{array}$ & $\begin{array}{l}\text { Las/los } \\
\text { estudiantes } \\
\text { evalúan el } \\
\text { trabajo de } \\
\text { los demás } \\
\text { equipos }\end{array}$ & $\begin{array}{l}\text { Crear un } \\
\text { espacio de } \\
\text { reflexión } \\
\text { acerca de los } \\
\text { desafios a la } \\
\text { hora de } \\
\text { evaluar el } \\
\text { trabajo de } \\
\text { otros equipos y } \\
\text { compararlo } \\
\text { con el trabajo } \\
\text { propio }\end{array}$ & $10 \%$ \\
\hline $\begin{array}{l}\text { Autoevaluación } \\
\text { individual (AI) }\end{array}$ & $\begin{array}{l}\text { Las/los } \\
\text { estudiantes } \\
\text { se asignan } \\
\text { un } \\
\text { calificación } \\
\text { individual } \\
\text { de acuerdo } \\
\text { a la } \\
\text { percepción } \\
\text { de su } \\
\text { desempeño }\end{array}$ & $\begin{array}{l}\text { Establecer } \\
\text { criterios de } \\
\text { confianza y } \\
\text { responsabilidad } \\
\text { entre el } \\
\text { docente y } \\
\text { las/los } \\
\text { estudiantes }\end{array}$ & $10 \%$ \\
\hline $\begin{array}{l}\text { Coevaluación } \\
\text { entre pares } \\
\text { (CP) }\end{array}$ & $\begin{array}{l}\text { Las/los } \\
\text { estudiantes } \\
\text { evaluan a } \\
\text { los } \\
\text { integrantes } \\
\text { de su } \\
\text { propio } \\
\text { grupo }\end{array}$ & $\begin{array}{l}\text { Incentivar una } \\
\text { participación } \\
\text { activa de todos } \\
\text { los integrantes }\end{array}$ & $20 \%$ \\
\hline $\begin{array}{l}\text { [1]Evaluación } \\
\text { de } \\
\text { presentación } \\
\text { oral del blog } \\
\text { (EB) }\end{array}$ & $\begin{array}{l}\text { El docente } \\
\text { evalúa el } \\
\text { blog, su } \\
\text { contenido y } \\
\text { la } \\
\text { presentación } \\
\text { que } \\
\text { realizan } \\
\text { las/los } \\
\text { estudiantes } \\
\text { del mismo }\end{array}$ & $\begin{array}{l}\text { Evaluar el } \\
\text { cumplimiento } \\
\text { de los objetivos } \\
\text { propuestos a } \\
\text { los equipos y } \\
\text { su adecuada } \\
\text { exposición del } \\
\text { contenido }\end{array}$ & $25 \%$ \\
\hline \multirow[t]{2}{*}{$\begin{array}{l}\text { Test individual } \\
\text { escrito (TI) }\end{array}$} & $\begin{array}{l}\text { Las/los } \\
\text { estudiantes } \\
\text { son } \\
\text { evaluados a } \\
\text { través de } \\
\text { un test } \\
\text { escrito igual } \\
\text { al empleado } \\
\text { en la } \\
\text { metodología } \\
\text { magistral }\end{array}$ & $\begin{array}{l}\text { Examinar el } \\
\text { logro y } \\
\text { comprensión } \\
\text { de conceptos } \\
\text { específicos } \\
\text { relevantes }\end{array}$ & $25 \%$ \\
\hline & & $\begin{array}{l}\text { Promedio Final } \\
(\mathrm{PF})\end{array}$ & $100 \%$ \\
\hline
\end{tabular}


Fuente: Elaboración propia con recopilación de material adaptado

de fuentes disponibles en literatura (Huebner, 2019; Stiggins, 2005).

Cabe destacar que se utilizó una rúbrica como instrumento de evaluación para todas las instancias, empleada por estudiantes y los grupos en los correspondientes contextos. Esta rúbrica se usó para las autoevaluaciones, las coevaluaciones y la presentación oral del blog (ver Tabla S2 del material suplementario). Dicha rúbrica considera todos los aspectos declarados en el resultado de aprendizaje final del curso, así como también el logro de las competencias de trabajo en equipo, su nivel de participación y el detalle de los contenidos abordados. La rúbrica, tal como se detalla en el material suplementario considera cuatro grandes categorías: presentación (20\%), competencias (20\%), contenido (40\%), estrategia (20\%). Estas categorías, los indicadores y sus ponderaciones fueron propuestas por personal docente y consensuadas con el estudiantado.

El test individual al término de la unidad se aplicó para verificar los conocimientos adquiridos por el alumnado; este test junto con la coevaluación de pares, permitió determinar la participación del estudiantado en el desarrollo del proyecto. Es importante mencionar que la estructura de las evaluaciones presentadas en la Tabla 2, corresponde a las evaluaciones finales; sin embargo, el trabajo fue retroalimentado por el docente desde la conformación de los equipos. Lo anterior debido a las características del blog, el cual permitió el intercambio y debate de ideas entre los participantes y el docente. Los blogs incluyeron una sección de sugerencias, en la cual el docente y las/los estudiantes al ingresar podían adjuntar un documento Word con correcciones, sugerencias o felicitaciones. En este sentido el blog fue una herramienta esencial no solo para la evaluación, sino también para la retroalimentación permanente del trabajo.

La estructura de las evaluaciones responde a las dos fases del Ciclo TBL, esto correspondiente al ciclo de aseguramiento del aprendizaje inicial y a las actividades de aplicación (Moraga y Soto, 2016) (ver Figura 1). Finalmente, se desarrolló un cuestionario para explorar la satisfacción del estudiantado respecto a la metodología, identificando si el trabajo propuesto logró satisfacer las expectativas grupales e individuales, respecto al ambiente de trabajo y aprendizajes alcanzados al desarrollar la actividad planteada.

En la Tabla 3 muestra las preguntas del cuestionario para evaluar el grado de satisfacción del estudiantado respecto a la metodología. Esta se compone de ocho reactivos en escala de estimación Likert donde 1: Totalmente en desacuerdo, 2: En desacuerdo, 3: De acuerdo, 4: Totalmente de acuerdo. Fue adaptada a partir de abundante revisión de literatura (Bouw, Gupta y Hincapie, 2015; Branson, Boss y Fowler, 2015; Haidet, Kubitz y McCormack, 2014; Vasan, DeFouw y Compton, 2009) y sometida a revisión por cuatro expertos. 
Guillermo Reyes-Torres, et al. Aprendizaje basado en eQuipos en un Curso de Ingeniería en EduCaCió...

TABLA 3

Cuestionario para evaluar el grado de satisfacción de la/el estudiante

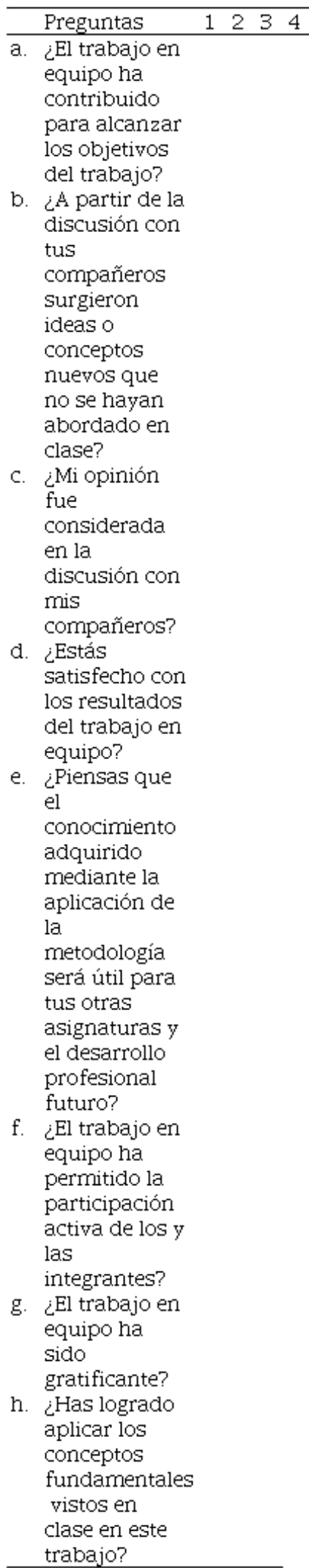


Fuente: Elaboración propia con material adaptado de Bouw et al.

(2015), Branson et al. (2015), Haidet et al. (2014) y Vasan et al. (2009)

\section{RESULTADOS, ANÁLISIS Y DISCUSIÓN}

\subsection{Estilos de aprendizaje}

Los resultados del test CHAEA o test de estilos de aprendizaje permite elaborar una serie de preguntas acerca de los hábitos y estilos de estudio del estudiantado, otorgándoles puntuación en cada uno de los cuatro estilos de aprendizaje de acuerdo con sus características personales, esto es: activo, reflexivo, teórico, pragmático (Alonso et al. 2007). El análisis estadístico de estas puntuaciones (basado en un análisis de la varianza entre estilos), permite definir cuál es el estilo de aprendizaje dominante en el/la estudiante, sin embargo, cuando dichas diferencias estadísticas no son determinantes se pueden encontrar combinaciones disímiles de estilos.

El resultado del test de clasificación de estilos de aprendizaje permitió subdividir los cursos en ocho grupos según los puntos obtenidos en el test CHAEA. Es importante resaltar que en la comunidad de investigación en educación existe cierto debate académico con respecto a la validez de los estilos de aprendizaje, lo anterior debido a que la principal hipótesis en la que se basa esta metodología es que la instrucción se debe proporcionar en un formato que coincida con las preferencias del alumnado y que además coincida con la mejor forma de evaluación posible de acuerdo con las mismas preferencias (Pashler, McDaniel, Rohrer y Bjork, 2008). Aun así existe evidencia de que estos estilos de aprendizaje facilitan el proceso formativo del estudiantado, enriquece el ambiente de trabajo y su motivación(Boyle, 2003; Kolb y Kolb, 2016).

En la Tabla 4 se puede observar los ocho grupos que resultaron, según las puntuaciones alcanzadas en el test de estilos de aprendizaje. Estos grupos fueron la base para conformar los equipos de trabajo de manera heterogénea. Esto permitió que en un solo grupo de trabajo no se concentraran solo integrantes con tendencia prioritaria a un estilo de aprendizaje: activo, pragmático, reflexivo y teórico. Se calcularon las puntuaciones de las/los estudiantes para cada estilo de aprendizaje, y se elaboró un análisis estadístico teniendo en cuenta los siguientes factores estadísticos. $M=$ valor promedio en la puntuación en cada estilo de aprendizaje, $S=$ desviación estándar del valor promedio, $\mathrm{V}=$ varianza del valor promedio. Se ejecutó un análisis considerando las puntuaciones de cada estudiante en cada estilo de aprendizaje y su ubicación con respecto a los factores estadísticos descritos anteriormente. Posterior a ello, se identificaron ocho diferentes tendencias resumidas en la Tabla 4. 
Guillermo Reyes-Torres, et al. Aprendizaje basado en equipos en un curso de Ingeniería en Educació...

TABLA 4

Estilos de aprendizaje según puntuación en el test CHAEA, años 2014 y 2016

\begin{tabular}{|c|c|c|}
\hline $\begin{array}{l}\text { Nombre } \\
\text { otorgado }\end{array}$ & \multirow[b]{2}{*}{$\begin{array}{l}\text { Descripción } \\
\text { del grupo }\end{array}$} & \multirow[t]{2}{*}{$\begin{array}{l}\text { Porcentaje } \\
\text { estudiantes }\end{array}$} \\
\hline G1 Homogéneo & & \\
\hline $\begin{array}{l}\text { Homogéneo } \\
\text { poco activo }\end{array}$ & $\begin{array}{l}\text { Perfiles } \\
\text { homogéneos } \\
\text { con alta } \\
\text { varianza } \\
(\mathrm{V}>2.5 \text { ). } \\
\text { Perfil activo } \\
\text { con } \\
\text { puntuación } \\
<\mathrm{M}-\mathrm{S}\end{array}$ & 25.0 \\
\hline G3 Reflexivo & $\begin{array}{l}\text { Perfiles con } \\
\text { alta } \\
\text { varianza } \\
(V>1.7) \text {. } \\
\text { Puntuación } \\
\text { en el perfil } \\
\text { reflexivo > } \\
M+S \text {, la } \\
\text { puntuación } \\
\text { en los } \\
\text { demás } \\
\text { perfiles es } \\
>M-S \text { y }< \\
M+S\end{array}$ & 17.5 \\
\hline $\begin{array}{ll}\text { Reflexivo } \\
\text { G4 }\end{array}$ & $\begin{array}{l}\text { Perfil igual } \\
\text { al G3. } \\
\text { Puntuación } \\
\text { en perfil } \\
\text { reflexivo > } \\
\text { M+S, pero } \\
\text { en uno de } \\
\text { los demás } \\
\text { estilos } \\
\text { posee una } \\
\text { calificación } \\
\text { menor a } \\
\text { M-S, por lo } \\
\text { tanto } \\
\text { poseen una } \\
\text { mayor } \\
\text { varianza } \\
(V>3)\end{array}$ & 15.0 \\
\hline G5 Activo & $\begin{array}{l}\text { Puntuación } \\
\text { estilo activo } \\
>\mathrm{M}+\mathrm{S} \text {, } \\
\text { puntuación } \\
\text { en estilo } \\
\text { teórico y lo } \\
\text { pragmático } \\
<\mathrm{M}-\mathrm{S}\end{array}$ & 15.0 \\
\hline $\begin{array}{ll}\text { G6 } & \begin{array}{l}\text { Reflexivo } \\
\text {-Teórico }\end{array}\end{array}$ & $\begin{array}{l}\text { Igual } \\
\text { tendencia } \\
\text { en estilos } \\
\text { teórico y } \\
\text { reflexivo }\end{array}$ & 2.5 \\
\hline G7 Pragmático & $\begin{array}{l}\text { Perfiles con } \\
\text { alta } \\
\text { varianza } \\
(V>1.7) \\
\text { Puntuación } \\
\text { en perfil } \\
\text { pragmático } \\
>M+S \text {, los } \\
\text { demás } \\
\text { perfiles se } \\
\text { encuentran } \\
\text { entre M-S y } \\
M+5\end{array}$ & 2.5 \\
\hline G8 Teórico & $\begin{array}{l}\text { Perfiles con } \\
\text { alta } \\
\text { varianza } \\
(V>1.7) \\
\text { Puntuación } \\
\text { perfil } \\
\text { teórico > } \\
M+S, \text { los } \\
\text { demás } \\
\text { perfiles se } \\
\text { encuentran } \\
\text { entre M-S y } \\
M+5\end{array}$ & 2.5 \\
\hline
\end{tabular}


Los resultados de la Tabla 4 muestran que el mayor porcentaje de las/los estudiantes no tiene un estilo de aprendizaje marcado o definido, esto ocurrió indistintamente para los cursos de 2014 y 2016. A partir de estos resultados y con el fin de conformar grupos de manera equilibrada, se establecieron algunas reglas heurísticas y por consenso entre las y los docentes de combinación, con el fin de que los grupos conformados presentaran estudiantes con la mayor variedad posible de estilos de aprendizaje. Las restricciones para integrar los equipos de trabajo fueron las siguientes: (1) estudiantes clasificados en los grupos G1 pueden formar grupos de trabajo con estudiantes con cualquier tipo de tendencia debido a que no poseen un estilo de aprendizaje preferente. Los integrantes identificados en el grupo G2 pueden integrar cualquier grupo y necesariamente con uno o más integrantes de los grupos G5 con el fin de reforzar el estilo activo dentro del equipo de trabajo. Los pertenecientes al grupo G5 pueden integrar equipos con todos los participantes, pero preferiblemente con integrantes del grupo G2 con el fin de dar una dinámica activa al grupo. Finalmente, los integrantes de los grupos G6, G7 y G8 pueden integrarse a un grupo de trabajo al azar, exceptuando un grupo que ya cuente con un miembro de dichos grupos (idealmente no integrar grupos que ya tengan representantes de los grupos G6, G7 y G8), con el propósito de no saturar los equipos con integrantes con enfoques solo teórico y/o pragmático.

Lo anterior, se desarrolló bajo la hipótesis de que un grupo de trabajo con integrantes representantes de todos los estilos de aprendizaje podría mostrar no solo un mejor rendimiento, si no también propiciar un mejor ambiente de trabajo, que de alguna manera emule las condiciones reales de trabajo en el ambiente laboral, dicha hipótesis es corroborada en los resultados mostrados con respecto al cuestionario la de satisfacción. En consideración a que la mayoría del estudiantado con que se trabajó no muestra estilos de aprendizaje definidos (ver Tabla 4), la conformación de grupos resultó compleja, aun así, se logró que en cada grupo existiese heterogeneidad en los estilos de aprendizaje de las/los estudiantes. De acuerdo a los comentarios expuestos durante las presentaciones orales del alumnado, se logró destacar que la presencia de este e con estilo de aprendizaje activo o pragmático fomentó la acción y ejecución de las tareas en grupo.

\subsection{Impacto cuantitativo}

Una vez conformados los grupos de trabajo y desarrollada la actividad junto con la construcción del blog, se aplicó evaluaciones cuantitativas, cuyos resultados promedio en los años 2014 y 2016, en escala de notas de 1 a 7 , se muestran en la Figura 3. 


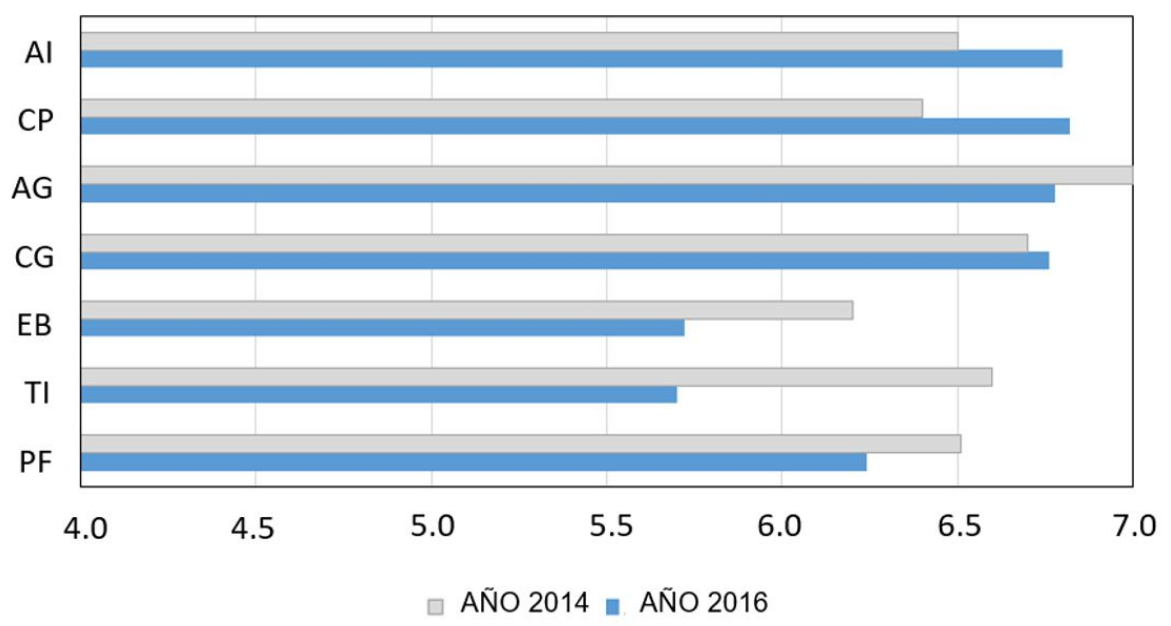

FIGURA 3.

Resultados de promedio de evaluaciones en escala de 1 a 7 puntos, años 2014 y 2016. Fuente: Elaboración propia

La Figura 3 detalla los resultados promedio para cada una de las evaluaciones detalladas en la Tabla 2. Los resultados de las evaluaciones cualitativas son en general altos, respecto a un estudio similar elaborado por Campillay (2015), donde las notas en promedio son de 4.5. En el presente trabajo las calificaciones son superiores a los 5.2 puntos por grupo, tanto en las versiones 2014 y 2016. Se observa, además, que las evaluaciones ejecutadas por el mismo estudiantado (autoevaluaciones y coevaluaciones individuales y grupales) tienen calificaciones, en general, más altas que las obtenidas en las evaluaciones supervisadas por la/ el docente a cargo. En este sentido las calificaciones son un $4 \%$ mayor en el caso de 2014 y un $18 \%$ mayor en el caso de 2016. Adicionalmente, en la Figura 3 se puede observar que las calificaciones efectuadas por el docente en el año 2016 fueron más bajas que las correspondientes calificaciones en el año 2014, esto provocó que las/los estudiantes se autoevaluaran y coevaluaran con calificaciones más altas, con el fin de compensar la calificación final.

Con el propósito de analizar con más detalle el desempeño de los grupos en cada tipo de evaluación, se desarrolla un desglose de estas notas para cada grupo. En la Figura 4 se muestran los resultados de la actividad para cada grupo.

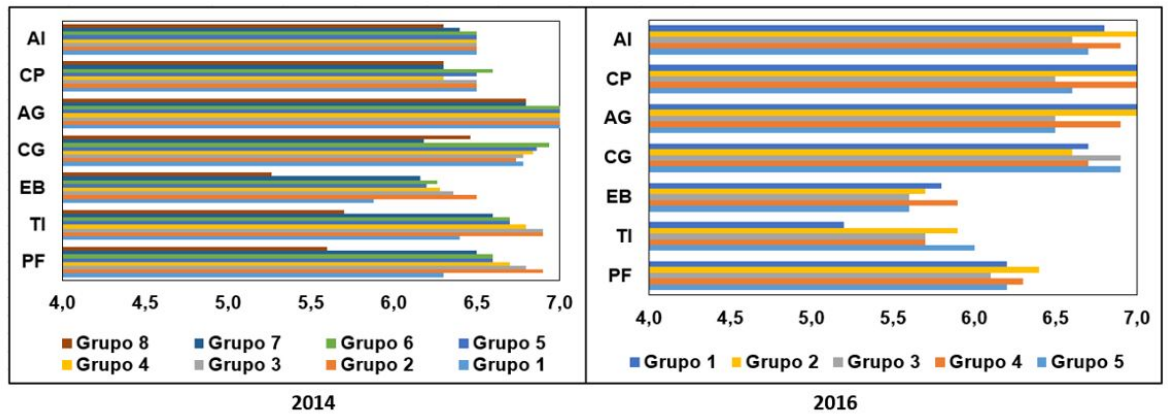

FIGURA 4.

Resultados grupales de evaluaciones en escala de 1 a 7 puntos, año 2016 Fuente: Elaboración propia

La Figura 4, muestra los resultados obtenidos para los grupos en el año 2016 y 2014, para la interpretación de los diferentes ítems de evaluación el lector debe observar la Tabla 2. Los resultados de la tabla revelan que las dos evaluaciones independientes ejecutadas por cada docente son inferiores a las notas, asignadas 
por el estudiantado, esto es: Presentación del Blog y Test individual, obteniendo una calificación promedio de 5.71 para el 2016 en el año 2014 ocurre lo contrario y lo cual parece indicar que el alumnado tiende a compensar su calificación. Así mismo las notas asignadas este, fueron en promedio de 6.79 para el 2016 indicando que cuando la/el docente asigna un menor valor a la nota, las/los estudiantes tienden en general a subir las calificaciones efectuadas por ellos, con el propósito de mantener su nota final promedio en un valor alto.

Con el fin de analizar un análisis completo de la efectividad de la metodología para tributar al incremento de las notas en el curso de Balances de materia y energía, en la Tabla 5 se resume el rendimiento de las/los estudiantes para todos los años (2014-2017) comparando las notas de esta actividad y las notas finales del curso para los años respectivos.

TABLA 5

Notas de la actividad desarrollada y notas finales del curso para los años (2014-2017)

\begin{tabular}{llllll}
\hline $\begin{array}{l}\text { Metodología } \\
\text { aplicada }\end{array}$ & Año & $\begin{array}{l}\text { Nota } \\
\text { actividad }\end{array}$ & $\begin{array}{l}\text { Nota } \\
\text { actividad } \\
\text { desv. }\end{array}$ & $\begin{array}{l}\text { Nota } \\
\text { curso }\end{array}$ & $\begin{array}{l}\text { Nota } \\
\text { curso }\end{array}$ \\
\hline TBL & 2014 & 6.5 & $(0.1)$ & 4.3 & $(0.9)$ \\
Magistral & 2015 & 6 & $(1)$ & 4.8 & $(0.7)$ \\
TBL & 2016 & 6.2 & $(0.1)$ & 4.9 & $(0.7)$ \\
Magistral & 2017 & 4.9 & $(1.9)$ & 4.4 & $(1.0)$ \\
\hline
\end{tabular}

Fuente: Elaboración propia

En la Tabla 5, los valores en paréntesis corresponden a las desviaciones estándar. De esta tabla se puede observar de manera más concreta que la metodología permitió obtener mejores notas para la actividad desarrollada con la metodología TBL $(6.5$ y 6.2) en los años 2014 y 2016, con respecto a las notas de $(6.0$ y 4.9) alcanzadas para los años 2015 y 2017 respectivamente. Adicionalmente para el año 2016 el curso alcanzo la nota final más alta (4.9) comparada con los demás años donde la misma nota fue $\geq 4.8$; y aunque no se alcanza a percibir una clara correlación entre el uso de la metodología y las notas finales del curso, si se puede evidenciar por parte de las/los estudiantes una mejor disposición al aprendizaje, tal como se evidencia en la siguiente sección.

\subsection{Retroalimentación de las/los estudiantes}

Como se mencionó anteriormente, la evaluación cualitativa del estudio consistió en un cuestionario de satisfacción estudiantil. La Figura 5 muestra los resultados para los años de aplicación 2014 y 2016, respectivamente. 


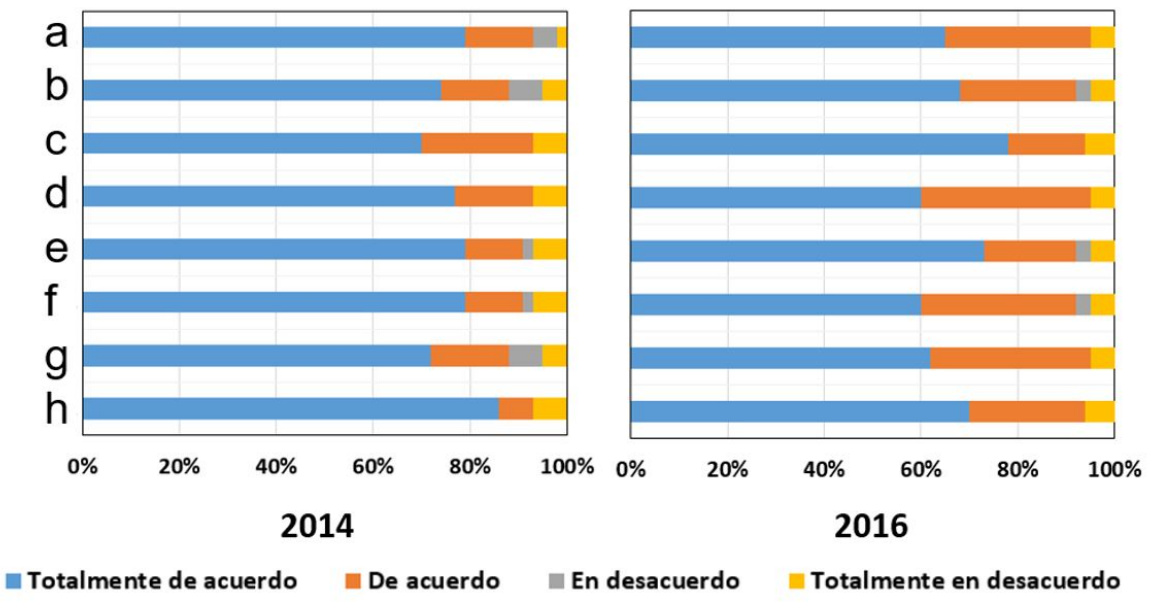

FIGURA 5

Resultados del cuestionario de percepción estudiantil años 2014 y 2016.

Nota: las preguntas son un extracto de las preguntas de la Tabla 2 ver pregunta según letra en dicha tabla. Fuente: Elaboración propia

Tras los resultados del cuestionario de percepción estudiantil mostrados en la Figura 5, es posible observar, que existe un alto porcentaje de conformidad respecto a la aplicación de la metodología, específicamente al trabajo en equipo, pues más del $60 \%$ de los grupos manifestaron que el trabajo en equipo fue gratificante, incluyó la opinión de todos los miembros y sirvió para alcanzar los objetivos propuestos (ver preguntas a, $\mathrm{d}, \mathrm{f}, \mathrm{g})$.

Por otra parte, para la versión de 2014 el porcentaje de conformidad es mayor (más del 70\% se mostró totalmente de acuerdo con estos aspectos), lo cual parece estar relacionado con un aumento en las expectativas de las/los estudiantes en la segunda versión del trabajo (teniendo como referente el trabajo desarrollado en 2014). En particular en la versión 2014, las/los estudiantes mostraron mayor conformidad con la metodología implementada, lo cual se visualiza a través de la obtención de un porcentaje más alto (>80\%) en la respuesta Totalmente de acuerdo con que la metodología sirvió efectivamente para conectar todos los conceptos vistos en clase (ver respuesta a pregunta h). Es importante destacar, que, aunque más estudiantes estuvieron totalmente de acuerdo en el año 2014, para el año 2016 la suma de estudiantes que estuvieron De acuerdo $y$ Totalmente de acuerdo, es mayor en todas las preguntas, por ejemplo, para la pregunta b, más del $95 \%$ de los grupos en el año 2016 estuvieron de acuerdo que la metodología propicio un diálogo en sus pares que permitió abordar aspectos nuevos no vistos en clase. Las respuestas del cuestionario aplicado entregaron información relevante respecto al nivel de participación y motivación de las/los estudiantes, corroborando que la conformación de grupos con integrantes que exhiben diferentes estilos de aprendizajes, facilitó el desarrollo de la actividad al interior de los grupos lo cual se confirmó desde los comentarios en las exposiciones orales y los resultados del cuestionario de percepción.

\section{Conclusiones}

La docencia en educación superior requiere cambios para responder a las necesidades actuales que demanda la sociedad del siglo XXI, la cual requiere de profesionales con competencias como el trabajo en equipo y colaborativo para resolver problemas en distintos ámbitos disciplinares. En este escenario este estudio constituye un aporte a la investigación sobre las metodologías activas en cursos para estudiantes de ingeniería en Educación Superior. Además, abre un camino de innovación, y una oportunidad para modificar el paradigma actual, en respuesta a las exigencias de los nuevos estudiantes y del mundo laboral. Dado el escenario actual de desarrollo tecnológico, la incorporación de apoyo de los recursos audiovisuales tiene 
una favorable acogida entre las/los estudiantes. En el caso de blogs, promueven la participación activa de las/los estudiantes, convirtiéndolos en protagonistas de su propio aprendizaje y las/los docentes actúan como facilitadores de ese aprendizaje, guiándolos constantemente. En este contexto los diferentes estilos de aprendizaje sirven como una plataforma de conformación de equipos más dinámicos e incluyentes, lo cual en comparación con los años que no se aplicó la metodología sirvió para evitar los comentarios de inconformidad por parte de las/los estudiantes acerca de la participación de los integrantes en el trabajo en equipo, y este aspecto es aún más relevante considerando que en los años que la metodología no fue aplicada dichos equipos fueron conformados libremente por las/los estudiantes sin tener en cuenta sus estilos de aprendizaje.

Del análisis de los resultados, se puede concluir que el estudiantado valoró positivamente la implementación del TBL, independiente al año de aplicación, pues la metodología ayuda a incrementar la motivación, el trabajo en equipo y el desempeño académico de las/los estudiantes. De igual forma la conformación de grupos de trabajo por integrantes con diferentes estilos de aprendizaje, impulsa la autogestión de roles y el liderazgo por parte de estudiantes con roles activos y pragmáticos, entregando resultados positivos en relación con el desempeño de los grupos y la participación del alumnado.

De la misma forma, se puede abstraer que el estudiantado aprende haciendo, lo cual se encuentra alineado con el constructivismo educativo que propone un paradigma en donde el proceso de enseñanza se percibe y se lleva a cabo como un proceso dinámico, participativo e interactivo del sujeto, de modo que el conocimiento sea una auténtica construcción del sujeto que aprende.

En conclusión, este estudio incentiva la aplicación del modelo como una posibilidad de superar algunas de las limitaciones de los modelos de enseñanza tradicionales, como las limitaciones de tiempo o la falta de motivación y el número de estudiantes. Este trabajo también contribuye a la creciente cantidad de publicaciones sobre la implementación de metodologías activas en el aprendizaje de Ingeniería Química en diferentes universidades latinoamericanas. Sin embargo, como posibles limitaciones del presente estudio, es importante mencionar que los resultados no pueden ser generalizados dado el alcance exploratorio de este proyecto. Por lo tanto, es recomendable el desarrollo futuro de estudios que puedan determinar con mayor contundencia los efectos que tiene esta metodología en el rendimiento académico más allá del grado de satisfacción expresado por los participantes y de sus notas promedio, considerando aspectos tales como la evolución de su rol en la construcción de conocimiento. Adicionalmente no se puede concluir que la metodología pueda mejorar las calificaciones finales en el curso; aunque si logra mejorar las calificaciones de la actividad desarrollada en particular. Lo anterior sugiere la necesidad de un nuevo trabajo involucrando todas las unidades temáticas que componen el curso de balances de materia y energía, considerando nuevas preguntas de investigación tales como: ¿Es posible lograr un mejor efecto empleando las mismas metodologías o una combinación de las mismas para todas las unidades temáticas? ¿Además de la elaboración del blog existen otras herramientas que se pudieran emplear con mayor impacto, tales como la elaboración de un video? ¿Es posible llevar a cabo la misma metodología en otros cursos del programa curricular de Ingeniería Civil Química, logrando mejores resultados? ¿Se pueden lograr mejores resultados si se entrena a las/los estudiantes acerca de las metodologías activas, de una manera temprana al inicio de la carrera universitaria? Estas y otras preguntas permitirían estandarizar y cuantificar de manera absoluta el impacto de esta y otras metodologías activas, no solo al interior del curso de balances de materia y energía sino también en otros cursos con contenidos y resultados de aprendizaje similares.

\section{ReFERENCIAS}

Alonso, C. , Gallego, D. y Honey, P. (2007). Los estilos de aprendizaje. Procedimientos y diagnóstico y mejora. Bilbao: Editorial Mensajero. 
Guillermo Reyes-Torres, et al. Aprendizaje basado en equipos en un curso de Ingeniería en Educació...

Bouw, J. W., Gupta, V. y Hincapie, A. L. (2015). Assessment of students' satisfaction with a student-led team-based learning course. Journal of Educational Evaluation for Health Professions, 12(23), 1-12.doi: https://doi.org/10 $.3352 /$ jeehp.2015.12.23

Boyle, E. A. (2003). Learning styles and academic outcome: The validity and utility of Vermunt. British Journal of Educational Psychology, 73(2), 267-290. doi: https://doi.org/10.1348/00070990360626976

Branson, S., Boss, L. y Fowler, D. L. (2015). Team-based learning: Application in undergraduate baccalaureate nursing education. Journal of Nursing Education and Practice, 6(4), 59-64. doi: https://doi.org/10.5430/jnep.v6n4p59

Bullard, L. G. y Felder, R. (2007). A Student-Centered Approach to Teaching Material and Energy Balances 2: Course Delivery and Assessment. Chemical Engineering Education, 41(2), 93-100. doi: https://doi.org/10.1002/9781 444398274.ch4

Bullard, L., Felder, R. y Raubenheimer, D. (2008). Effects of active learning on student performance and retention. En Proceedings. Annual Conference of the American Society for Engineering Education. Pittsburgh

Campillay, M. A. N. (2015). Analysis of impact of methodology activates and heuristic learning in subjects of engineering. Actualidades Investigativas En Educación, 15, 1-16.

Felder, R. M., Brent, R. y Carolina, N. (2009). Active learning: an introduction. ASQ Higher Education Brief, 2(4), 1-5.

Freeman, S., Eddy, S. L., McDonough, M., Smith, M. K., Okoroafor, N., Jordt, H. y Wenderoth, M. P. (2014). Active learning increases student performance in science, engineering, and mathematics. Proceedings of the National Academy of Sciences, 111 (23), 8410-8415. doi: https://doi.org/10.1073/pnas.1319030111

García, F. C. (2014). Usos específicos de los blogs como herramienta educativa. Nómadas. Revista Crítica de Ciencias Sociales y Jurídicas, 32(2), 1-9.

González, A., Montero, S. y Laza, T. (2006). Uso de wikis para la realización de trabajos colaborativos en el aula. En XIV Jornadas de ASEPUMA y II Encuentro Internacional. España.

Haidet, P., Kubitz, K. y McCormack, W. T. (2014). Analysis of the Team-Based Learning Literature: TBL Comes of Age.J Excell Coll Teach., 25(3-4), 303-333. doi: https://doi.org/10.1002/cncr.27633.Percutaneous

Hemant, N. y Stefanek, G. (2016). Continuous Evaluation of Student Class Performance Using Group-based, In-class Quizzes. En ASEE'S 123rd Annual Conference and Exposition. New Orleans: AIChe.

Honey, P. y Mumford, A. (2000). The Learning Styles Helper's Guide. Reino Unido: Peter Honey Publications.

Huebner, T. (2019). What Research Says About.../Balanced Assessment. Educational Leadership, 37(3), 85-86.

Keith, J. M., Silverstein, D. L., Visco, D. P. y Bullard, L. G. (2010). Ideas to consider for new chemical engineering educators: senior design. Chemical Engineering Education, 44(4), 306-317.

Kennedy, D., Hyland, Á. y Ryan, N. (2014). Writing and Using Learning Outcomes\#: A Practical Guide Writing and Using Learning Outcomes\#: a Practical Guide. Cork: University College Cork

Kolb Y, A. y Kolb A, D. (2016). Learning Styles and Learning Spaces: Enhancing Experiential Learning in Higher Education. Academy of Management Learning y Education, 4(2), 193-212. doi: https://doi.org/10.5465/AM LE.2005.17268566

Liberatore, M. W. (2011). Improved Student Achievement Using Personalized Online Homework for a Course in Material and Energy Balances. Chemical Engineering Education, 45(3), 184-190.

Mason, G. S., Shuman, T. R. y Cook, K. E. (2013). Comparing the effectiveness of an inverted classroom to a traditional classroom in an upper-division engineering course. IEEE Transactions on Education, 56(4), 430-435. doi: htt ps://doi.org/10.1109/TE.2013.2249066

Maure, L. M. y Marimón, O. G. (2014). Examining the role of college student's approach to Math. Educational Research and Reviews, 9(19), 761-770.

Michaelsen, L. K., Knight, A. B. y Fink, L. D. (2004). Team-Based Learning: A Transformative Use of Small Groups in College Teaching. Sterling. Estados Unidos: Stylus.

Moraga, D. y Soto, J. (2016). TBL-Aprendizaje Basado en Equipos. Estudios Pedagógicos, 42(2), 437-447.

Pashler, H., McDaniel, M., Rohrer, D. y Bjork, R. (2008). Learning Styles: Concepts and Evidence. Psychological Science in the Public Interest, 9(3), 105-119. doi: https://doi.org/10.1111/j.1539-6053.2009.01038.x 
Pérez, A. M. y Poveda, P. (2008). Efectos del Aprendizaje Cooperativo en la Adaptación Escolar. Revista de Investigación Educativa, 26(1), 79-34. Recuperado de http://www.redalyc.org/articulo.oa?id=283321884005

Prince, M. (2004). Does Active Learning Work? A Review of the Research. Journal of Engineering Education, 93(3), 223-231. Recuperado de http://proxy.library.upenn.edu

Prince, M. J. y Felder, R. M. (2006). Inductive teaching and learning methods: Definitions, comparisons, and research bases. Journal of Engineering Education, 95(2), 123-138. doi: https://doi.org/10.1002/j.2168-9830.2006.tb00 884.x

Silverstein, D. L., Bullard, L. G. y Vigeant, M. A. (2018). How We Teach: Material and Energy Balances. En AIChe (Ed.), How We Teach\#: Material and Energy Balances (pp. 1-14). Kentucky, Estados Unidos: UK College of Engineering Surveys.

Stiggins, R. (2005). From Formative Assessment to Assessment for Learning: A Path to Success in Standards-Based Schools. The Phi Delta Kappan, 87(4), 324-328. Recuperado de http://www.jstor.org/stable/20441998

Tweddell, S. J. (2017). Introducing Team-Based Learning in a Pharmacy Curriculum\#: a Qualtiative Study of Staff and Student Experiences.(Thesis Doctor). University of Huddesfield, Huddersfield.

Vasan, N. S., DeFouw, D. O. y Compton, S. (2009). A survey of student perceptions of team-based learning in anatomy curriculum: Favorable views unrelated to grades, Anat Sci Ed, 2(4), 150-155. doi: https://doi.org/10.1002/ase .91

Zheng, B., Niiya, M. y Warschauer, M. (2015). Wikis and collaborative learning in higher education. Technology, Pedagogy and Education, 24(3), 357-374. doi: https://doi.org/10.1080/1475939X.2014.948041

\section{Notas}

[1] Esta evaluación y el test individual son elaborados por el docente, las demás evaluaciones son efectuadas por estudiantes, con el fin de lograr una participación activa y significativa de todas las partes.

\section{BY-NC-ND}

